\title{
Relationship between levels of serum gastric inhibitory polypeptide (GIP), soluble interleukin-2 receptor (sIL-2R), and soluble triggering receptor expressed on myeloid cells-1 (sTREM-1) and disease condition and prognosis of patients with severe acute pancreatitis
}

\author{
Li Zeng ${ }^{1,2,3 \#}$, Fanjie Xi ${ }^{1,2 \#}$, Yang Yang ${ }^{2,4}$, Lu Guo ${ }^{2,4}$, Honglin $\mathrm{Hu}^{2,5}$, Qian Yang ${ }^{6}$, Qunhua Ma ${ }^{2,4}$ \\ ${ }^{1}$ Department of Nursing, Sichuan Provincial People's Hospital, University of Electronic Science and Technology of China, Chengdu, China; \\ ${ }^{2}$ Chinese Academy of Sciences, Sichuan Translational Medicine Research Hospital, Chengdu, China; ${ }^{3}$ Department of Nursing, Chengdu Qing \\ Cheng Mt. Hospital, Chengdu, China; ${ }^{4}$ RICU \& MICU, Sichuan Provincial People's Hospital, University of Electronic Science and Technology of \\ China, Chengdu, China; ${ }^{5}$ Department of Cancer Center, Sichuan Provincial People's Hospital, University of Electronic Science and Technology of \\ China, Chengdu, China; ${ }^{6}$ College of Nursing, Chengdu Medical College, Chengdu, China \\ Contributions: (I) Conception and design: L Zeng, F Xi; (II) Administrative support: Q Yang, Q Ma; (III) Provision of study materials or patients: All \\ authors; (IV) Collection and assembly of data: All authors; (V) Data analysis and interpretation: All authors; (VI) Manuscript writing: All authors; (VII) \\ Final approval of manuscript: All authors. \\ \#These authors contributed equally to this work. \\ Correspondence to: Qian Yang. College of Nursing, 601 Tian Hui Road, Rongdu Avenue, Jinniu District, Chengdu, China. Email: yq1811793@163.com; \\ Qunhua Ma. RICU \& MICU, 32 West Road Section 2, First Ring Road, Chengdu, China. Email: mqh1770813@163.com.
}

\begin{abstract}
Background: To explore the relationship between levels of serum gastric inhibitory polypeptide (GIP), soluble interleukin-2 receptor (sIL-2R), and soluble triggering receptor expressed on myeloid cells-1 (sTREM-1) and the disease condition and prognosis in patients with severe acute pancreatitis (SAP).

Methods: A total of 52 patients with SAP (SAP group) and 50 patients with mild acute pancreatitis (MAP group) admitted to Sichuan Provincial People's Hospital, University of Electronic Science and Technology of China between April 2017 and December 2019 were included in the present study. A further 50 people who had received a healthy physical examination during the same period constituted the healthy control group. The levels of serum GIP, sIL-2R, and sTREM-1 were measured. The levels of serum GIP, sIL2R, and sTREM-1 were compared among the SAP, MAP and healthy control groups, and the severity of disease (Ranson scoring system) was compared between the SAP and MAP groups. Pearson correlation analysis was used to analyze the correlation between the levels of serum GIP, sIL-2R, and sTREM-1 with the Ranson scores in the SAP group. A receiver operating characteristic (ROC) curve was drawn to evaluate the predictive efficacy of serum GIP, sIL-2R, and sTREM-1 on prognosis.

Results: The levels of serum GIP, sIL-2R, and sTREM-1 in the SAP and MAP groups were higher than those in the healthy control group, and the levels in the SAP group were higher than those in the MAP group. The Pearson correlation analysis showed that the levels of serum GIP, sIL-2R, and sTREM-1 in the SAP group were positively correlated with Ranson scores. The levels of serum GIP, sIL-2R, and sTREM-1 in the survival group were lower than those in the deceased group. The ROC curve showed that the best cut-off values of serum GIP, sIL-2R, and sTREM-1 in predicting prognostic survival were $167.040 \mathrm{pg} / \mathrm{mL}$, $70.840 \mathrm{pg} / \mathrm{mL}$, and $128.325 \mathrm{ng} / \mathrm{mL}$, respectively.

Conclusions: The levels of serum GIP, sIL-2R, and sTREM-1 are closely related to the severity of illness in patients with SAP and can be used as reference indicators for assessing the onset of SAP and predicting prognosis.
\end{abstract}


Keywords: Severe acute pancreatitis (SAP); gastric inhibitory polypeptide (GIP); soluble interleukin-2 receptor (sIL-2R); soluble triggering receptor expressed on myeloid cells-1 (sTREM-1); disease condition; prognosis

Submitted Apr 22, 2021. Accepted for publication Jun 01, 2021.

doi: 10.21037/apm-21-1231

View this article at: https://dx.doi.org/10.21037/apm-21-1231

\section{Introduction}

Acute pancreatitis is a common clinical acute abdomen disease, and most cases are mild and self-limiting. In severe cases, pancreatic hemorrhage and necrosis may occur, which may be secondary to peritonitis and shock, resulting in a poor prognosis and high mortality (1). Severe acute pancreatitis (SAP) has rapid clinical changes, and the early assessment of the severity of the disease and timely intervention measures are therefore vitally important to improve prognosis and reduce the mortality rate (2). In the early stage of SAP, inflammatory factors play a key role in the initial systemic inflammatory response, and as the disease progresses, secondary infections and even multiple organ failure can occur. In addition, damage to intestinal functioning also occurs early in the course of SAP, and intestinal bacterial translocation is another important cause of later infection. It has been shown that damage to the intestinal mucosal barrier function is closely related to SAP (3). Soluble interleukin-2 receptor (sIL-2R) and soluble myeloid cell trigger receptor (sTREM-1) are related indicators of inflammation, and gastrointestinal peptide (GIP) is related to gastrointestinal functioning. This study aims to explore the relationship between the above three serum indicators and the disease condition and prognosis of SAP patients. We present the following article in accordance with the STARD reporting checklist (available at http://dx.doi.org/10.21037/apm-21-1231).

\section{Methods}

\section{Research participants}

We selected 52 SAP patients (SAP group) and 50 patients with mild acute pancreatitis (MAP group) who were admitted to Sichuan Provincial People's Hospital, University of Electronic Science and Technology of China between April 2017 and December 2019. All patients agreed to participate in this study and signed an informed consent form. The study was conducted in accordance with the Declaration of Helsinki (as revised in 2013). This study was approved by the Sichuan Provincial People's Hospital, University of Electronic Science and Technology of China (No. 20170318). A further 50 healthy patients who had completed a physical examination in the same time period were selected as the healthy control group. The inclusion criteria for the SAP and MAP groups were as follows: a diagnosis complying with the relevant criteria for the diagnosis and classification of acute pancreatitis in the "Guidelines for the Diagnosis of Acute Pancreatitis" of the Chinese Society of Gastroenterology (4); patients who had been admitted to the hospital within 48 hours of onset; and patients who were treatment naïve. All patients were required to give informed consent for this study, including voluntary cooperation with the relevant examinations. The exclusion criteria were as follows: patients who died within 48 hours of admission; those with suspected or diagnosed malignant tumors; those who had chronic pancreatitis; those who had a history of long-term immunosuppressant or hormone drug use; and those who had incomplete clinical and follow-up data. Healthy control subjects were excluded if they had a history of rheumatism, tumors, or infectious diseases. In the SAP group, there were 30 males and 22 females aged $31-79$ years old (average age $54.27 \pm 7.49$ years); the etiology of the group consisted of 10 idiopathic cases, 40 biliary cases, and 2 alcoholic cases. In the MAP group, there were 28 males and 22 females aged 33-78 years old (average age 54.03 \pm 7.92 years); the etiology of the group consisted of 7 idiopathic cases, 41 biliary cases, and 2 alcoholic cases. In the healthy control group, there were 27 males and 23 females aged 29-77 years old (average age $53.99 \pm 8.13$ years). There were no significant differences in the general information of the three groups $(\mathrm{P}>0.05)$.

\section{Index detection method}

On admission to hospital, $4 \mathrm{~mL}$ of fasting cubital venous blood was collected from all study participants, centrifuged for $5 \mathrm{~min}$ at 3,000 $\mathrm{r} / \mathrm{min}$, and the supernatant was then separated and stored in a refrigerator at $-70{ }^{\circ} \mathrm{C}$ for later use. An enzyme-linked immunosorbent assay was used to determine the levels of serum GIP, sIL-2R, and 
Table 1 Comparison of levels of serum GIP, sIL-2R, and sTREM-1 among the three groups $(\bar{x} \pm s)$

\begin{tabular}{lcccc}
\hline Time & Case number & GIP $(\mathrm{pg} / \mathrm{mL})$ & $\mathrm{sIL}-2 \mathrm{R}(\mathrm{pg} / \mathrm{mL})$ & $\mathrm{sTREM}-1(\mathrm{ng} / \mathrm{nL})$ \\
\hline SAP group & 52 & $164.55 \pm 11.51^{\mathrm{ab}}$ & $54.56 \pm 9.91^{\mathrm{ab}}$ & $92.19 \pm 12.74^{\mathrm{ab}}$ \\
MAP group & 50 & $137.69 \pm 10.07^{\mathrm{a}}$ & $35.49 \pm 9.74^{\mathrm{a}}$ & $63.04 \pm 10.12^{\mathrm{a}}$ \\
Healthy control group & 50 & $104.29 \pm 6.12$ & $15.72 \pm 4.51$ & $36.03 \pm 8.62$ \\
F value & - & 510.278 & 268.496 & 353.501 \\
P value & - & $<0.001$ & $<0.001$ & $<0.001$ \\
\hline
\end{tabular}

Compared with healthy control group, ${ }^{\mathrm{a}} \mathrm{P}<0.05$; compared with MAP group, ${ }^{\mathrm{b}} \mathrm{P}<0.05$. GIP, gastric inhibitory polypeptide; sIL-2R, soluble interleukin-2 receptor; sTREM-1, soluble triggering receptor expressed on myeloid cells-1; SAP, severe acute pancreatitis; MAP, mild acute pancreatitis; $P$ value, probability; F value, oscillator strength.

sTREM-1. Analysis was conducted by the IMMULITE chemiluminescence enzyme immunoassay analyzer (Diagnostic Products Corporation, USA). The test kits were purchased from the Diagnostic Products Corporation (USA), and the instructions were strictly followed.

\section{Assessment of severity of illness (5)}

The Ranson scoring system was used for evaluation. The scoring content covers five indicators: age at admission, blood sugar, white blood cell count, aspartate aminotransferase, and lactate dehydrogenase. Seven indicators assess blood pressure, body fluid loss, and hematocrit reduction. In addition, blood calcium, blood urea nitrogen, alkali loss, and oxygen content are assessed within 48 hours of admission. The total score is 12 points (the higher the score, the more severe the pancreatitis).

\section{Treatment and prognosis evaluation}

Patients in the SAP group were treated symptomatically according to the relevant standards in the treatment guidelines. According to the 28-day survival outcomes, patients were further divided into a survival group and a deceased group.

\section{Observation indicators}

The levels of serum GIP, sIL-2R, and sTREM-1 were compared between the SAP group, the MAP group, and the healthy control group. The severity of disease (Ranson scoring system) was compared between the SAP and the MAP groups. Pearson correlation was used to analyze the correlation between the serum GIP, sIL-2R, and sTREM-1 levels and the Ranson score in the SAP group. The levels of serum GIP, sIL-2R, and sTREM-1 in the survival group and the deceased group were compared. A receiver operating characteristic (ROC) curve was used to evaluate the predictive power of serum GIP, sIL-2R, and sTREM-1 on prognosis.

\section{Statistical analysis}

SPSS 22.0 (Armonk, NY, USA) was used for the data analysis. The measurement data were normally distributed and described by the mean \pm standard deviation $(\bar{x} \pm s)$. The independent $t$ test was used for two-group comparisons, and the analysis of variance was used to compare multiple groups. Enumeration data were expressed by $\mathrm{n}(\%)$, and the $\chi^{2}$ test was used for between-group comparisons. The Pearson rank correlation coefficient was used for the correlation analysis. The predictive value of the ROC curve evaluated indicators for prognosis. A $\mathrm{P}$ value $<0.05$ was considered to be statistically significant.

\section{Results}

Comparison of serum GIP, sIL-2R, and sTREM-1 levels in the three groups

As shown in Table 1, the levels of serum GIP, sIL-2R, and sTREM-1 in the SAP and MAP groups were higher than those in the healthy control group. The levels of serum GIP, sIL-2R, and sTREM-1 were higher in the SAP group than in the MAP group $(\mathrm{P}<0.05)$.

\section{Comparison of the Ranson scores between the SAP and MAP groups}

The Ranson scores of the SAP group were higher than 
those of the MAP group $(\mathrm{P}<0.05)$ (see Table 2).

\section{Correlation analysis between the serum GIP, sIL-2R, and sTREM-1 levels and the Ranson scores}

Pearson correlation analysis showed that the levels of serum GIP, sIL-2R, sTREM-1 in the SAP group were positively correlated with the Ranson scores (all $\mathrm{P}<0.05)$ (see Table 3).

\section{Comparison of serum GIP, sIL-2R, and sTREM-1 levels in the survival and deceased groups}

As shown in Table 4, the levels of serum GIP, sIL-2R, and sTREM-1 in the survival group were lower compared with those in the deceased group (all $\mathrm{P}<0.05$ ).

Table 2 Comparison of Ranson scores between the SAP and MAP groups $(\bar{x} \pm s)$

\begin{tabular}{lcc}
\hline Group & Case number & Ranson score (points) \\
\hline SAP group & 52 & $5.65 \pm 1.79$ \\
MAP group & 50 & $2.31 \pm 1.03$ \\
$t$ value & - & 11.490 \\
$P$ value & - & $<0.001$ \\
\hline
\end{tabular}

SAP, severe acute pancreatitis; MAP, mild acute pancreatitis; $P$ value, probability; $t$-test, Student's $t$ test.
The predictive power of serum GIP, sIL-2R, and sTREM-1 on prognostic survival

As shown in Figure 1 and Table 5, the ROC curve showed that the best truncation value of serum GIP, sIL-2R, and sTREM-1 for predicting prognostic survival were $18.10 \mathrm{pg} / \mathrm{mL}$, $3.17 \mathrm{pg} / \mathrm{mL}$, and $283.77 \mathrm{ng} / \mathrm{mL}$, respectively. The corresponding areas under the ROC curve were 0.861 , $0.821,0.783$, respectively.

\section{Discussion}

As SAP progresses, it can be accompanied by pancreatic hemorrhage, necrosis infection, and even multi-system organ failure. The condition changes rapidly, the case fatality rate exceeds $20 \%$, and the prognosis is poor (6). Therefore, timely assessment of the condition of SAP patients and timely effective intervention measures are critical to improve prognosis and reduce mortality.

Recent research suggests that the pathogenesis and disease progression of SAP are closely related to inflammation and microcirculation dysfunction $(7,8)$. Systemic inflammation can stimulate monocytes to release chemokines, which in turn induce a phosphorylation cascade, which intensifies the inflammatory response, activates leukocytes and endothelial cells, and further increases the release of related inflammatory factors (9). Previous studies have proposed the "cytokine theory" in the

Table 3 Correlation between levels of serum GIP, sIL-2R, and Ranson scores

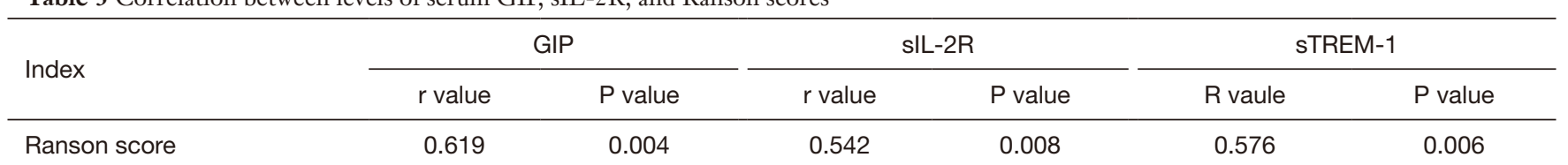

GIP, gastric inhibitory polypeptide; sIL-2R, soluble interleukin-2 receptor; sTREM-1, soluble triggering receptor expressed on myeloid cells-1; P value, probability; $r$ value, right value.

Table 4 Comparison of levels of serum GIP, sIL-2R, and sTREM-1 between the survival group and deceased group $(\bar{x} \pm \mathrm{s})$

\begin{tabular}{lcccc}
\hline Group & Case number & GIP $(\mathrm{pg} / \mathrm{mL})$ & $\mathrm{slL}-2 \mathrm{R}(\mathrm{pg} / \mathrm{mL})$ & $\mathrm{sTREM}-1(\mathrm{ng} / \mathrm{nL})$ \\
\hline Survival group & 42 & $152.79 \pm 14.27$ & $40.42 \pm 10.13$ & $71.23 \pm 14.02$ \\
Deceased group & 10 & $218.63 \pm 7.63$ & $113.95 \pm 6.07$ & $180.22 \pm 7.29$ \\
$t$ value & - & 14.046 & 21.933 & 23.705 \\
$P$ value & - & $<0.001$ & $<0.001$ & $<0.001$ \\
\hline
\end{tabular}

GIP, gastric inhibitory polypeptide; sIL-2R, soluble interleukin-2 receptor; sTREM-1, soluble triggering receptor expressed on myeloid cells-1; $\mathrm{P}$ value, probability; $t$-test, Student's $t$ test. 


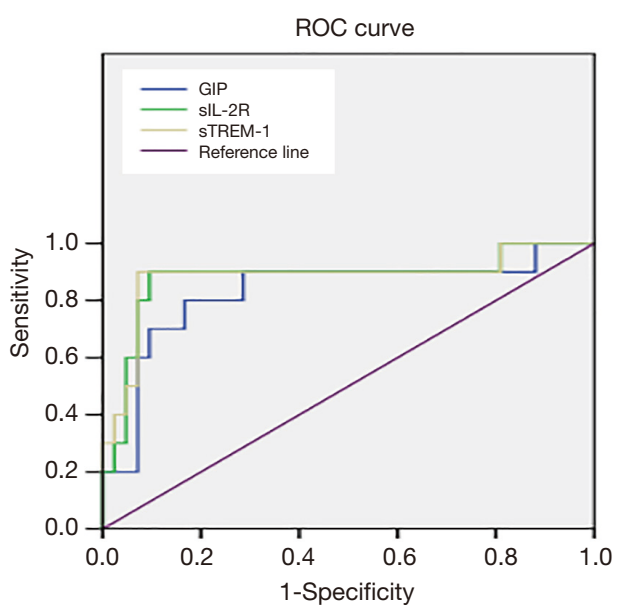

Figure 1 ROC curves of serum GIP, sIL-2R, and sTREM-1 in predicting prognostic survival. ROC, receiver operating characteristic; GIP, gastric inhibitory polypeptide; sIL-2R, soluble interleukin-2 receptor; sTREM-1, soluble triggering receptor expressed on myeloid cells-1.

pathogenesis of SAP (10). sIL-2R is an immunosuppressive inhibitor of the synthesis and secretion of monocytes and activated $\mathrm{T}$ cells. It has a regulatory effect on the function of the interleukin-2 (IL-2)/IL-2R system and competes with IL-2R to bind IL-2, inhibit the IL-2 autocrine, and then reduce the IL-2 immune response $(11,12)$. Related studies have confirmed that the upregulation of sIL-2R expression is accompanied by decreased $\mathrm{B}$ cell immune function (13). Another study found that SAP showed significant immunosuppression in the early stage of the disease, but this gradually returned to normal with disease improvement, indicating that the development of SAP is related to changes in the body's immune system (14). sTREM-1 is a soluble immune protein superfamily receptor expressed by monocytes, macrophages, and neutrophils. It is involved in the triggering, mediation, and amplification of inflammatory responses, can promote the expression of pro-inflammatory cytokines, and inhibits the release of anti-inflammatory factors. The formation is called the "inflammatory waterfall effect" $(15,16)$. The results of this study showed that the levels of serum sIL-2R and sTREM-1 in the SAP group and the MAP group were higher compared with the healthy control group. The levels of serum sIL-2R and sTREM-1 in the SAP group were higher than those of the MAP group, indicating that sIL$2 \mathrm{R}$ and sTREM-1 are related to SAP progression. sIL$2 \mathrm{R}$ and sTREM-1 mediate complex immune damage, and
Table 5 Diagnostic value of serum GIP, sIL-2R, and sTREM-1 on prognostic survival

\begin{tabular}{lcl}
\hline Index & Best truncation value & AUC \\
\hline GIP & 167.040 & 0.829 \\
SIL-2R & 70.840 & 0.879 \\
STREM-1 & 128.325 & 0.883 \\
\hline
\end{tabular}

GIP, gastric inhibitory polypeptide; sIL-2R, soluble interleukin-2 receptor; sTREM-1, soluble triggering receptor expressed on myeloid cells -1 .

in the early course of SAP, their expression is increased, causing the release of inflammatory factors from pancreatic alveolar cells to increase sharply, triggering an inflammatory cascade. Inflammation spreads from local to systemic organs, affecting the prognosis (17). This study showed that the levels of serum sIL-2R and sTREM-1 in the SAP group were positively correlated with the Ranson score, and the levels of serum sIL-2R and sTREM-1 in the survival group were lower compared with the deceased group. Further analysis of the ROC curve found that both serum sIL-2R and sTREM-1 have a high efficacy in predicting prognosis and survival, confirming their value in assessing disease and predicting prognosis.

With a deeper understanding of the pathogenesis of SAP, scholars have found that pancreatitis is closely related to changes in gastrointestinal functioning (18). In SAP patients, abdominal pain, bloating, and other gastrointestinal dysfunction-related symptoms are clinically common. In severe cases, gastrointestinal bleeding may occur, and the mortality rate is increased (19). Studies have confirmed that SAP may affect gastrointestinal functioning in different ways (20). On the one hand, the retroperitoneal space has a blocking effect on the spread of pancreatic inflammation, but when the pressure of the retroperitoneum is at a high level for a long time, abdominal cavity syndrome can occur, causing complications such as intestinal necrosis; on the other hand, SAP can stimulate gastrointestinal hormone secretion, which affects gastrointestinal functioning (21). GIP is an essential gastrointestinal hormone, which has inhibitory effects on gastric peristalsis, emptying, and gastric acid secretion (22). According to the results of this study, the levels of serum GIP in the SAP group and MAP group were higher compared with the healthy control group. The levels of serum GIP in the SAP group were higher than the MAP group. The Pearson correlation analysis showed that the levels of serum GIP in 
the SAP group were positively correlated with the Ranson scores, indicating serum GIP upregulation is closely related to deterioration in SAP. Previous studies have confirmed that gastrointestinal motility disorder can occur following the initial remission of SAP, and gastrointestinal hormones such as motilin (MTL) are correlated with the prognosis of SAP patients $(23,24)$. However, there are few studies on the association between GIP and SAP prognosis. The 28-day survival results of this study showed that the levels of serum GIP in the survival group patients were lower than those in the deceased group. Additional ROC curve analysis showed that the level of serum GIP has a high predictive power for the prognostic survival of patients, indicating that this index is closely related to the prognosis of SAP.

In summary, the levels of serum GIP, sIL-2R, and sTREM-1 are closely related to the severity of SAP and can be used as reference indicators for assessing the onset of SAP and predicting prognosis. Early detection is essential in guiding the formulation of clinical interventions.

\section{Acknowledgments}

Funding: None.

\section{Footnote}

Reporting Checklist: The authors have completed the STARD reporting checklist. Available at https://dx.doi. org/10.21037/apm-21-1231

Data Sharing Statement: Available at https://dx.doi. org/10.21037/apm-21-1231

Conflicts of Interest: All authors have completed the ICMJE uniform disclosure form (available at https://dx.doi. org/10.21037/apm-21-1231). The authors have no conflicts of interest to declare.

Ethical Statement: The authors are accountable for all aspects of the work in ensuring that questions related to the accuracy or integrity of any part of the work are appropriately investigated and resolved. All patients agreed to participate in this study and signed an informed consent form. The study was conducted in accordance with the Declaration of Helsinki (as revised in 2013). This study was approved by the Sichuan Provincial People's Hospital, University of Electronic Science and Technology of China (No. 20170318).
Open Access Statement: This is an Open Access article distributed in accordance with the Creative Commons Attribution-NonCommercial-NoDerivs 4.0 International License (CC BY-NC-ND 4.0), which permits the noncommercial replication and distribution of the article with the strict proviso that no changes or edits are made and the original work is properly cited (including links to both the formal publication through the relevant DOI and the license). See: https://creativecommons.org/licenses/by-nc-nd/4.0/.

\section{References}

1. Rashid N, Sharma PP, Scott RD, et al. All-Cause and Acute Pancreatitis Health Care Costs in Patients With Severe Hypertriglyceridemia. Pancreas 2017;46:57-63.

2. Ding L, Deng F, Yu C, et al. Portosplenomesenteric vein thrombosis in patients with early-stage severe acute pancreatitis. World J Gastroenterol 2018;24:4054-60.

3. Liang HY, Chen T, Yan HT, et al. Berberine ameliorates severe acute pancreatitis-induced intestinal barrier dysfunction via a myosin light chain phosphorylation-dependent pathway. Mol Med Rep 2014;9:1827-33.

4. Leppäniemi A, Tolonen M, Tarasconi A, et al. 2019 WSES guidelines for the management of severe acute pancreatitis. World J Emerg Surg 2019;14:27.

5. Harputluoglu MMM, Ozbek M, Demirel U, et al. Relationship between nucleotide-binding oligomerization domain-containing protein 2 variants and severity of acute pancreatitis. Acta Gastroenterol Belg 2019;82:285-90.

6. Portelli M, Jones CD. Severe acute pancreatitis: pathogenesis, diagnosis and surgical management. Hepatobiliary Pancreat Dis Int 2017;16:155-9.

7. Zhang J, Yu WQ, Wei T, et al. Effects of Short-PeptideBased Enteral Nutrition on the Intestinal Microcirculation and Mucosal Barrier in Mice with Severe Acute Pancreatitis. Mol Nutr Food Res 2020;64:e1901191.

8. Dumnicka P, Maduzia D, Ceranowicz P, et al. The Interplay between Inflammation, Coagulation and Endothelial Injury in the Early Phase of Acute Pancreatitis: Clinical Implications. Int J Mol Sci 2017;18:354.

9. Gomes CA, Di Saverio S, Sartelli M, et al. Severe acute pancreatitis: eight fundamental steps revised according to the 'PANCREAS' acronym. Ann R Coll Surg Engl 2020;102:555-9.

10. Meyer A, Kubrusly MS, Salemi VM, et al. Severe acute pancreatitis: a possible role of intramyocardial cytokine production. JOP 2014;15:237-42. 
11. Teodorczyk-Injeyan JA, Sparkes BG, Lalani S, et al. IL-2 regulation of soluble IL-2 receptor levels following thermal injury. Clin Exp Immunol 1992;90:36-42.

12. Degiannis D, Hornung N. Rapamycin inhibits the in vitro release of soluble interleukin-2 receptor by activated peripheral blood mononuclear cells (PBMC) independently of the mode of activation. Int J Immunopharmacol 1995;17:593-6.

13. Aguilar-Santelises M, Loftenius A, Ljungh C, et al. Serum levels of helper factors (IL-1 alpha, IL-1 beta and IL6), T-cell products (sCD4 and sCD8), sIL-2R and beta 2-microglobulin in patients with B-CLL and benign $\mathrm{B}$ lymphocytosis. Leuk Res 1992;16:607-13.

14. Ueda T, Takeyama Y, Yasuda T, et al. Immunosuppression in patients with severe acute pancreatitis. J Gastroenterol 2006;41:779-84.

15. Bassyouni IH, Fawzi S, Gheita TA, et al. Clinical Association of a Soluble Triggering Receptor Expressed on Myeloid Cells-1 (sTREM-1) in Patients with Systemic Lupus Erythematosus. Immunol Invest 2017;46:38-47.

16. Bostanci N, Thurnheer T, Aduse-Opoku J, et al. Porphyromonas gingivalis regulates TREM-1 in human polymorphonuclear neutrophils via its gingipains. PLoS One 2013;8:e75784.

Cite this article as: Zeng L, Xi F, Yang Y, Guo L, Hu H, Yang Q, Ma Q. Relationship between levels of serum gastric inhibitory polypeptide (GIP), soluble interleukin-2 receptor (sIL-2R), and soluble triggering receptor expressed on myeloid cells-1 (sTREM-1) and disease condition and prognosis of patients with severe acute pancreatitis. Ann Palliat Med 2021;10(6):6786-6792. doi: 10.21037/apm-21-1231
17. Palmiere C, Egger C. Usefulness of pericardial and pleural fluids for the postmortem diagnosis of sepsis. J Forensic Leg Med 2014;28:15-8.

18. Jin M, Zhang HM, Chen XF, et al. Evaluation and Early Diagnosis of Gastrointestinal Failure in Acute Pancreatitis. Zhongguo Yi Xue Ke Xue Yuan Xue Bao 2020;42:47-54.

19. Agarwala R, Rana SS, Sharma R, et al. Gastrointestinal Failure Is a Predictor of Poor Outcome in Patients with Acute Pancreatitis. Dig Dis Sci 2020;65:2419-26.

20. Chen S, Wang L, Gao X, et al. Characterization of serum irisin in patients with severe acute pancreatitis. Eur J Gastroenterol Hepatol 2019;31:985-91.

21. Li XY, He C, Zhu Y, et al. Role of gut microbiota on intestinal barrier function in acute pancreatitis. World J Gastroenterol 2020;26:2187-93.

22. Yasuda K, Seino Y. Gastric inhibitory polypeptide (GIP) and GIP receptor (GIPR). Nihon Rinsho 1996;54:1078-82.

23. Gold-Smith FD, Singh RG, Petrov MS. Elevated Circulating Levels of Motilin are Associated with Diabetes in Individuals after Acute Pancreatitis. Exp Clin Endocrinol Diabetes 2020;128:43-51.

24. Fox JE. Motilin--an update. Life Sci 1984;35:695-706.

(English Language Editor: D. Fitzgerald) 